\title{
On the variation of carbon abundance in galaxies and its implications
}

\author{
D. Romano ${ }^{1}$, M. Franchini ${ }^{2}$, V. Grisoni ${ }^{3,4,2}$, E. Spitoni ${ }^{5}$, F. Matteucci ${ }^{4,2,6}$ and C. Morossi ${ }^{2}$ \\ 1 INAF, Astrophysics and Space Science Observatory, Via Gobetti 93/3, 40129 Bologna, Italy \\ e-mail: donatella.romano@inaf.it \\ 2 INAF, Osservatorio Astronomico di Trieste, Via Tiepolo 11, 34131 Trieste, Italy \\ 3 SISSA, Via Bonomea 265, 34136 Trieste, Italy \\ ${ }^{4}$ Dipartimento di Fisica, Sezione di Astronomia, Università di Trieste, Via Tiepolo 11, 34131 Trieste, Italy \\ 5 Stellar Astrophysics Centre, Department of Physics and Astronomy, Aarhus University, Ny Munkegade 120, 8000 Aarhus C, \\ Denmark \\ ${ }^{6}$ INFN, Sezione di Trieste, Via Valerio 2, 34127 Trieste, Italy
}

Received 17 March 2020 / Accepted 11 May 2020

\begin{abstract}
The trends of chemical abundances and abundance ratios observed in stars of different ages, kinematics, and metallicities bear the imprints of several physical processes that concur to shape the host galaxy properties. By inspecting these trends, we get precious information on stellar nucleosynthesis, the stellar mass spectrum, the timescale of structure formation, the efficiency of star formation, as well as any inward or outward flows of gas. In this paper, we analyse recent determinations of carbon-to-iron and carbon-to-oxygen abundance ratios in different environments (the Milky Way and elliptical galaxies) using our latest chemical evolution models that implement up-to-date stellar yields and rely on the tight constraints provided by asteroseismic stellar ages (whenever available). A scenario where most carbon is produced by rotating massive stars, with yields largely dependent on the metallicity of the parent proto-star clouds, allows us to fit simultaneously the high-quality data available for the local Galactic components (thick and thin discs) and for microlensed dwarf stars in the Galactic bulge, as well as the abundance ratios inferred for massive elliptical galaxies. Yet, more efforts are needed from both observers and theoreticians in order to base these conclusions on firmer grounds.
\end{abstract}

Key words. nuclear reactions, nucleosynthesis, abundances - galaxies: abundances - galaxies: evolution

\section{Introduction}

Carbon is one of the most abundant elements in the Universe and a powerful key to understanding stellar and galactic structure and evolution.

In stellar interiors, carbon is an important contributor to the opacity (e.g., Marigo 2002, and references therein) and, as a catalyst in the CNO cycle, it affects the energy generation that accompanies thermonuclear fusion (Bethe 1939, Burbidge et al. 1957). The ratio of the abundances of the two stable isotopes of carbon, ${ }^{12} \mathrm{C}$ and ${ }^{13} \mathrm{C}$, measured at the surface of stars evolved off the main sequence and compared to evolutionary model predictions, informs us about the occurrence and strength of nuclear burning and mixing processes along the giant branches (Charbonnel 1994, Gratton et al. 2000, Lagarde et al. 2019; Maas et al.2019). On the other hand, the anticorrelation between $\mathrm{C}$ and $\mathrm{N}$ abundances observed in main-sequence globular cluster (GC) stars cannot be attributed to internal stellar processes (Cannon et al. 1998; Harbeck et al. 2003); rather, it adds up to the many peculiarities of stellar populations in GCs (see Gratton et al. 2019, for a recent review) that still wait for a satisfactory explanation (Bastian \& Lardo 2018). Carbon detections also prove useful for the characterisation of the atmospheres of exoplanets (e.g., Vidal-Madjar et al. 2004).

On the scale of galaxies, carbon abundances can be used to constrain the timescales of structure formation (e.g., Matteucci
2012) and to pin down the shape of the galaxy-wide stellar initial mass function (gwIMF, Jeřábková et al. 2018) in objects where direct estimates are impossible (Romano et al.2017, Zhang et al. 2018). Carbon also traces the distribution of cool gas in local and distant galaxies via molecular and atomic fine structure line emission, thus providing the necessary link between stellar and star formation rate densities in the Universe (Carilli \& Walter 2013). Carbon monoxide further traces giant molecular outflows in bright quasar hosts and starburst-dominated galaxies (e.g., Feruglio et al. 2010, Cicone et al. 2014), shedding light on the ultimate driver of such gigantic outward motions of processed gas.

From the incomplete list of topics above it is already apparent that carbon is an element of paramount importance in many research fields of contemporary astrophysics. In particular, a firm understanding of the origin and evolution of carbon in the Milky Way remains a crucial step towards the development of theoretical models aimed at explaining $\mathrm{C}$ abundance measurements in other systems: indeed, such models should always be tested and comply with local data first. In this respect, it is worth recalling that the stellar yields of carbon (i.e., the amounts of newlyproduced $\mathrm{C}$ that dying stars eject in their surroundings) are not firmly established yet and, therefore, the fractional contribution to the carbon abundance from different sources is still under debate (see, e.g., Romano et al. 2017, 2019, for a recent reappraisal of this problem). 
Nowadays, the flood of data secured by large modern spectroscopic surveys, such as the Gaia-ESO Public Spectroscopic Survey (GES, Gilmore et al. 2012), the Apache Point Observatory Galactic Evolution Experiment (APOGEE, Majewski et al. 2017), and the GALactic Archaeology with HERMES (GALAH, De Silva et al. 2015), provides the raw material for sophisticated analyses involving thousands of stars that belong to different Galactic components. As a result, statistically significant trends of abundance ratios with metallicity can be obtained for different stellar populations, which can then be meaningfully compared to the predictions of theoretical models.

In this paper we deal with the origin and evolution of carbon in galaxies. First, we compare the results of our Galactic chemical evolution (GCE) models to the $[\mathrm{C} / \mathrm{Fe}]$ versus $[\mathrm{Fe} / \mathrm{H}]$ and $[\mathrm{C} / \mathrm{O}]$ versus $[\mathrm{O} / \mathrm{H}]$ abundance ratios obtained by Amarsi et al. (2019) from a reanalysis of literature data for a sample of nearby late-type stars including departures from local thermodynamic equilibrium (LTE) and corrections for three-dimensional (3D) stellar atmospheres. The theoretical results are confronted also with median sequences of $[\mathrm{C} / \mathrm{X}]$ versus $[\mathrm{X} / \mathrm{H}]$ (where $\mathrm{X}=$ $\mathrm{Fe}, \mathrm{O}$ and/or $\mathrm{Mg}$ ) derived for two larger samples of solar neighbourhood stars, one selected from the GALAH second data release (DR2, Griffith et al.2019) and the other from the GES fifth internal data release (iDR5, Franchini et al. 2020). The different data sets are further compared to each other (see next section). Second, we comment on $[\mathrm{C} / \mathrm{X}]$ determinations in other environments, focusing on the evolution of carbon at high metallicities. Lastly, basing on the comparison between model predictions and observations, we discuss the fractional contribution to $\mathrm{C}$ enrichment that is coming from different stellar polluters.

The paper is organised as follows. In Sect. 2 we describe the data used for the comparison with the outputs of the models that, in turn, are portrayed in Sect. 3. Sect. 4 presents our results, which are further discussed in Sect.5. Finally, Sect.6reports our conclusions.

\section{Observational data}

\subsection{High-resolution spectroscopy of solar vicinity stars}

For the purpose of comparison with our model results, we consider the carbon, oxygen and iron abundances homogeneously derived by Amarsi et al. (2019) for a sample of $187 \mathrm{~F}$ and $\mathrm{G}$ dwarfs belonging to the Galactic disc and halo components. The fiducial abundance set is based on a 3D non-LTE line formation analysis, which leads to a reduced scatter and trends at low metallicities that deviate markedly from those obtained in the classical 1D LTE approximation (see Amarsi et al. 2019, their Figs. 11 to 13). Consistently with previous work by Nissen et al. (2014), it is found that thick-disc stars have $[\mathrm{C} / \mathrm{Fe}]$ and $[\mathrm{O} / \mathrm{Fe}]$ ratios higher than thin-disc stars over the full metallicity range spanned by the observations, with the possible exception of the highest metallicities; in the $[\mathrm{C} / \mathrm{O}]$ versus $[\mathrm{O} / \mathrm{H}]$ plane, instead, thin-disc stars display the highest ratios (see, however, Bensby \& Feltzing 2006, for different results).

When high-resolution $(R \geq 40000)$, high signal-to-noise $(S / N \geq 150)$ spectra are available, as it is the case of the studies mentioned above, very small random errors can be associated to single abundance measurements. A significant increase in the sample size (by one or more orders of magnitude) further allows for the definition of tight average trends.

Griffith et al. (2019) have applied a median trend analysis to more than 70000 stars from GALAH DR2; among these, $\sim 12000$ high-metallicity $([\mathrm{Fe} / \mathrm{H}]>-0.4 \mathrm{dex})$, hot $(5500<$
$T_{\text {eff }} / \mathrm{K}<6500$ ) stars have $\mathrm{C}$ detections (see Buder et al. 2019). The sample is separated in high- $\alpha$ and low- $\alpha$ stars; these are renamed low-Ia and high-Ia stars, respectively, following common wisdom that a lower/higher level of Fe enrichment from Type Ia supernovae (SNeIa) has to be expected in the thick/thin disc because of its faster/slower evolution (time-delay model, Tinsley 1980, see also Matteucci \& Greggio 1986, their Fig. 3). We take advantage of the median low-Ia and high-Ia sequences defined in the $[\mathrm{C} / \mathrm{X}]$ versus $[\mathrm{X} / \mathrm{H}]^{1}$ planes by Griffith et al. (2019) to validate our models.

Moreover, we consider the $[\mathrm{C} / \mathrm{Fe}]$ versus $[\mathrm{Fe} / \mathrm{H}]$ and $[\mathrm{C} / \mathrm{Mg}]$ versus $[\mathrm{Mg} / \mathrm{H}]$ average behaviours for thick- and thin-disc stars selected on a chemical basis from GES iDR5 by Franchini et al. (2020). These authors have re-analysed spectra taken with UVES in the setup centred at $580 \mathrm{~nm}(R \simeq 47000)$ and derived carbon abundances from atomic lines for 2133 FGK stars. The final abundance trends are not corrected for 3D non-LTE effects since, at a first approximation, these prove not to affect the results significantly in the metallicity range spanned by GES observations (see Franchini et al. 2020, their Sect. 2.2.2).

In Fig. 1 1 we plot for thin- and thick-disc stars the $[\mathrm{C} / \mathrm{Fe}]$ (left panel) and $[\mathrm{C} / \mathrm{Mg}]$ (right panel) abundance ratios versus $[\mathrm{Fe} / \mathrm{H}]$ and $[\mathrm{Mg} / \mathrm{H}]$, respectively, using abundance estimates from targeted observations by several authors (triangles: Nissen \& Schuster 2010, Nissen et al. 2014, squares: Bensby \& Feltzing |2006; Bensby et al. |2014; circles: 3D non-LTE abundance estimates by Amarsi et al. 2019); the corresponding trends from GES iDR5 (continuous lines: Franchini et al. 2020) and from GALAH DR2 (dashed lines: Griffith et al.2019) are also shown. Red and blue colours are adopted to identify thin- and thick-disc stars, respectively. From Fig. 1 we can clearly observe significant differences between the trends from the two surveys including systematic offsets. The GES trends are lower than the GALAH ones and neither of them agree with the loci of the individual points, even if they are characterised by a large spread. Actually, the comparison between abundances derived by different studies is always difficult because several factors (e.g., different resolution and $S / N$, atmospheric parameters uncertainties, different methods of analysis, etc.) may introduce large systematic differences.

A common approach in literature is to remove at least systematic offsets by introducing zero-point corrections so that thin-disc stars with $[\mathrm{Fe} / \mathrm{H}] \simeq 0$ (or $[\mathrm{Mg} / \mathrm{H}] \simeq 0$ ) also have solar chemical composition (i.e., $[\mathrm{X} / \mathrm{Fe}]$ or $[\mathrm{X} / \mathrm{Mg}] \simeq 0$ ). As an example, Griffith et al. (2019) apply zero-point offsets to their GALAH DR2 data points and trends so that stars with $[\mathrm{Fe} / \mathrm{Mg}]=[\mathrm{Mg} / \mathrm{H}] \simeq 0$ also have $[\mathrm{X} / \mathrm{Mg}] \simeq 0$. We adopted a similar approach and computed for each group of data shown in Fig. 1 zero-point offsets in order to have $[\mathrm{C} / \mathrm{Fe}]$ and $[\mathrm{C} / \mathrm{Mg}] \simeq 0$ for thin-disc stars with $[\mathrm{Fe} / \mathrm{H}]$ or $[\mathrm{Mg} / \mathrm{H}] \simeq 0$, respectively. Figure 2 shows the same comparison as in Fig. 1 but after applying these zero-point offsets. From the left panel we can see that the agreement between the offset [C/Fe] GES and GALAH trends is good but for the metal-poor thin-disc stars, where the GALAH trend falls at higher $[\mathrm{C} / \mathrm{Fe}]$ than the GES one. The latter seems in better agreement with the area covered by individual points than the former, but the large scatter of individual abundance ratios prevents us to draw a clear conclusion.

The spread in the thin and thick GES [C/Fe] abundance ratios is shown by the pale red and blue areas, respectively, with boundaries corresponding to the 10th to 90th percentiles. The quite large vertical extension of these two areas $(\simeq \pm 0.2 \mathrm{dex})$

${ }^{1} \mathrm{X}=\mathrm{Fe}, \mathrm{O}$, and $\mathrm{Mg}$. 

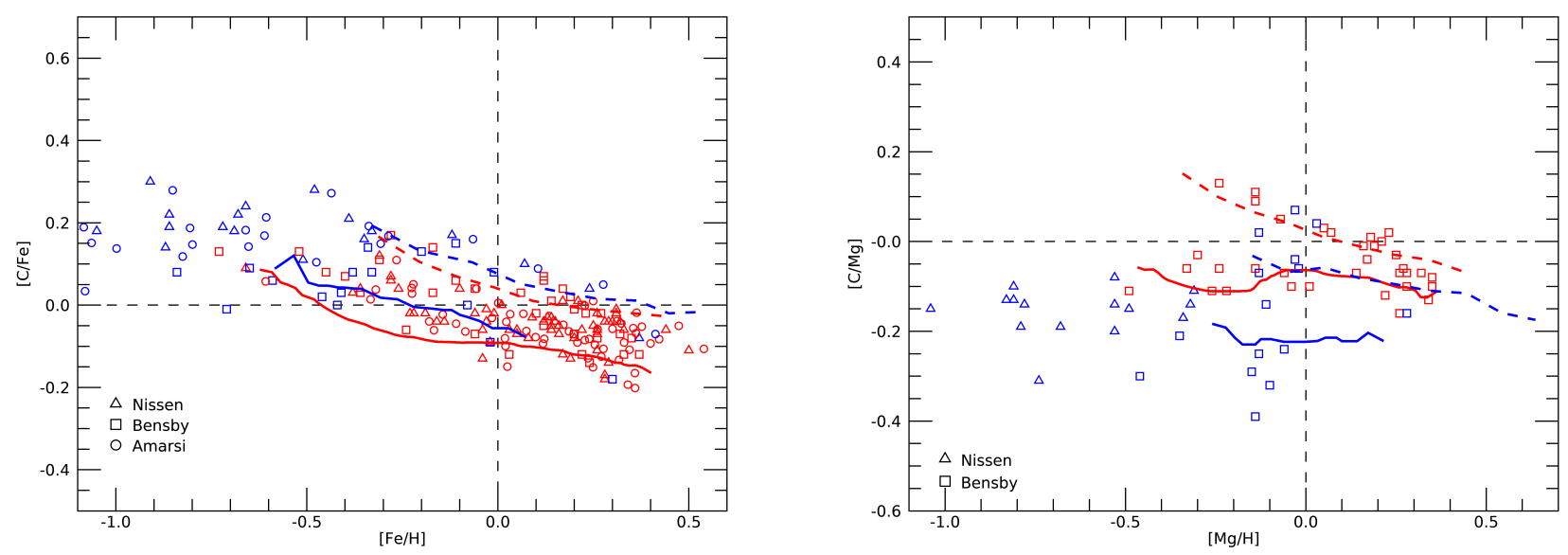

Fig. 1. Carbon-to-iron (left panel) and carbon-to-magnesium (right panel) abundance ratios as functions of $[\mathrm{Fe} / \mathrm{H}]$ and $[\mathrm{Mg} / \mathrm{H}]$, respectively. The red and blue symbols represent abundance estimates from targeted observations of thin- and thick-disc stars, respectively (triangles: Nissen \& Schuster 2010, Nissen et al. 2014, squares: Bensby \& Feltzing 2006, for C; Bensby et al. 2014 for Mg; circles: Amarsi et al. 2019). The red and blue lines represent trends of abundance ratios of thin- and thick-disc stars, respectively, derived from: (i) nearly 1300 thin-disc and 100 thick-disc dwarf stars from GES iDR5, selected on the bases of chemical criteria (continuous lines; Franchini et al. 2020); (ii) more than 12000 stars from GALAH DR2, divided into high-Ia and low-Ia sequences (dashed lines; Griffith et al.2019). No zero-point offsets are applied to the data.
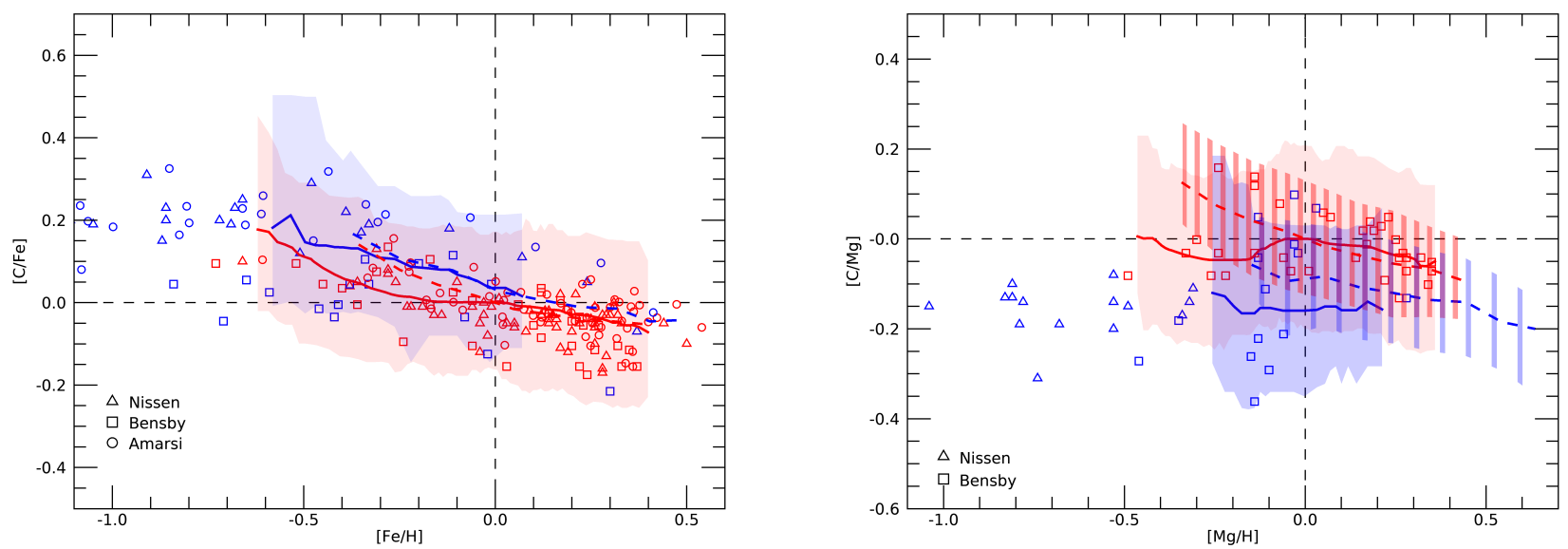

Fig. 2. Same as Fig. 1 but with zero-point offsets (see text) applied to the data. Pale red and blue areas show the spread in thin- and thick-disc GES data within boundaries corresponding to the 10th and 90th percentiles. In the right panel, dashed red and blue areas show the analogous spread in the GALAH thin- and thick-disc data, respectively.

may come from a combination of uncertainties in the derived abundance ratios (due to the relatively low $S / N$ of some of the GES spectra) and of a possible cosmic scatter. On the other hand, the fact that also the individual points, which are affected by much smaller uncertainties, span similar wide areas seems to indicate that the cosmic scatter may be significant for both thinand thick-disc stars. A similar result was found for $[\mathrm{O} / \mathrm{Fe}]$ in the Galactic disc by Bertran de Lis et al. (2016) who found that the square root of the star-to-star cosmic variance in $[\mathrm{O} / \mathrm{Fe}]$ ratio at a given metallicity is about $0.03-0.04$ dex in both the thin and thick disc.

In the right panel of Fig. 2 we plot the same quantities as in the left panel but for $[\mathrm{C} / \mathrm{Mg}]$ versus $[\mathrm{Mg} / \mathrm{H}]$. Moreover, we plot in this panel as red and blue vertical shaded regions the spread areas, between the 10th and 90th percentiles, covered by GALAH data of thin- and thick-disc stars as provided to us by E. Griffith (private communication). It can be seen that the GALAH spread areas are comparable in size with the GES ones taking also into account the different number of stars in the two samples. Also in this diagram the scatter of the individual points is similar to those of the surveys, thus supporting the presence of a cosmic scatter in $[\mathrm{C} / \mathrm{Mg}]$ as in $[\mathrm{C} / \mathrm{Fe}]$.

Even if by applying zero-point offsets the discrepancies between GES and GALAH trends showed in the right panel of Fig. 1 are reduced, significant differences still remain in Fig. 2. In particular, $[\mathrm{C} / \mathrm{Mg}]$ GES trends are flatter than GALAH ones and in better agreement with the loci of the individual points, especially for the thick-disc stars. It may be worthwhile pointing out that flat trends of $[\mathrm{C} / \mathrm{Mg}]$ are expected if $\mathrm{C}$ and $\mathrm{Mg}$ are mainly produced by the same progenitors (see discussion in Sect. 5. 


\subsection{Microlensed $\mathrm{G}$ dwarfs in the Galactic bulge}

The targets of the high-resolution studies reviewed in the previous section are all main-sequence or subgiant stars. The original carbon content of their atmospheres is not altered by internal evolutionary processes and as such, they provide a fossil record of the $\mathrm{C}$ enrichment histories of the different Galactic components they belong to. However, they are faint and probe mostly the immediate surroundings of the Sun.

In principle, high-resolution spectra can be obtained also for dwarf stars in the Galactic bulge, if they are optically magnified during gravitational microlensing events. The stars can then be analysed the same way as control sample analogs in the solar neighbourhood, thus enabling a fully differential comparison between different Galactic populations (e.g., Bensby et al. 2010). In practice, to the best of our knowledge carbon abundances have been derived only for three microlensed dwarfs in the bulge ${ }^{2}$, namely, MOA-2006-BLG-099S (Johnson et al. 2008), MOA2008-BLG-310S and MOA-2008-BLG-311S (Cohen et al. 2009).

\subsection{Early-type galaxies}

Along with the Galactic bulge, massive elliptical galaxies are the stellar systems where one expects to see the cleanest signs of nucleosynthesis at high metallicity.

From an analysis of unresolved stellar populations in nearly 4000 early-type galaxies from the Sloan Digital Sky Survey (SDSS, York et al. 2000), Johansson et al. (2012) find that $[\mathrm{C} / \mathrm{Mg}]$ is about solar for the most massive systems, while [C/O] is slightly higher, namely $[\mathrm{C} / \mathrm{O}] \sim 0.05$ (see also Conroy et al. 2014). It is suggested that similar values of the abundance ratios imply a lower limit for the formation timescale of massive ellipticals of about $0.4 \mathrm{Gyr}$, which is the lifetime of a $3 \mathrm{M}_{\odot}$ star (the underlying hypothesis is that in order to explain the observed ratios intermediate-mass stars must contribute their newly-produced carbon). In Sect. 5] we revise this assertion in the light of updated stellar nucleosynthesis and chemical evolution calculations.

\section{Chemical evolution models}

In this work, we adopt the following chemical evolution models:

1. The two-infall chemical evolution model for the Milky Way halo and discs, originally developed by Chiappini et al. (1997, 2001). This model divides the Galactic disc in several concentric annuli $2 \mathrm{kpc}$ wide that evolve independently, without exchanges of matter between them (but see Spitoni et al. 2015). The inner halo and thick disc are assumed to form fast, in less than $1 \mathrm{Gyr}$, out of a first episode of accretion of virgin gas. During these earlier evolutionary stages, the star formation proceeds very efficiently and turns a large fraction of the available gas into stars. Eventually, a critical gas density threshold is reached below which the star formation halts. Later on, a second infall episode, delayed $1 \mathrm{Gyr}$ with respect to the previous one, starts replenishing the disc with fresh gas and star formation is reignited. The

\footnotetext{
2 T. Bensby and collaborators have collected a statistically significant sample of 91 microlensed dwarf and subgiant stars in the bulge (Bensby et al. 2017, and references therein). They provide stellar ages and abundances for several elements, but do not encompass carbon: that's a bummer!
}

thin disc thence forms on longer timescales that are functions of the Galactocentric distance (Matteucci \& François 1989; Romano et al. 2000). This scheme has been recently revised by Spitoni et al. (2019) who have taken into account the constraints on stellar ages provided by asteroseismology and fixed the delay time for the second infall to $4.3 \mathrm{Gyr}$, i.e., much longer than assumed in the original formulation of the model. Here we show results obtained for the solar neighbourhood in either case.

2. The parallel model proposed by Grisoni et al. (2017) that envisages distinct formation sequences for the thick and thin discs (see also Grisoni et al. 2019, 2020). In the parallel model, the two discs form independently on different timescales out of two separate infall episodes. In this work we consider only the formation of the local discs (i.e., within $1 \mathrm{kpc}$ from the Sun), but the model has been extended to other Galactocentric distances (Grisoni et al. 2018).

3. The reference model for the Galactic bulge by Matteucci et al. (2019), which assumes that the majority of the stars in the so-called 'classical bulge' are old and form quickly in a very efficient starburst. These assumptions, jointly to the adoption of a gwIMF flatter than the one derived for the solar vicinity, guarantee that the stellar metallicity and age distributions, as well as the $[\mathrm{Mg} / \mathrm{Fe}]$ ratios, are well reproduced by the model (Matteucci et al. 2019, see also Matteucci \& Brocato 1990, Matteucci et al. 1999).

4. The model for the prototype massive elliptical galaxy described in Romano et al. (2017, 2019). According to these authors, the most massive early-type galaxies form stars intensively while hidden in heavy dust curtains at high redshifts, where they show up as submillimeter-bright galaxies. After reaching a stellar mass of about $2 \times 10^{11} \mathrm{M}_{\odot}$, their residual gas is swept away by the cleaning action of largescale outflows triggered by SN explosions and AGN activity. The galaxies evolve passively since then. Similarly to what is done for the Galactic bulge, a top-heavy gwIMF is assumed, which guarantees a good fit to the CNO isotopic ratios measured in submillimeter galaxies (Romano et al.2017; Zhang et al. 2018).

\subsection{Basic assumptions}

The chemical evolution models adopted in this study track the evolution of the chemical composition of the interstellar medium (ISM) in different galaxies and/or Galactic components. They deal with several elements, from the lightest ones emerging from Big Bang nucleosynthesis (Romano et al. 2003) to the heaviest ones synthesised by uncommon astrophysical sites, like magneto-rotational driven $\mathrm{SNe}$, and/or through rare events, as compact binary mergers (Matteucci et al. 2014, Cescutti et al. 2015. Grisoni et al. 2020).

Cold gas of primordial chemical composition is accreted at an exponentially decreasing rate. For the two-infall model,

$\frac{\mathrm{d} \mathscr{M}_{\text {inf }}(t)}{\mathrm{d} t}=c_{1} \mathrm{e}^{-t / \tau_{1}}+c_{2} \mathrm{e}^{-\left(t-t_{\max }\right) / \tau_{2}}$,

where $\mathscr{M}_{\text {inf }}(t)$ is the mass accreted at time $t, \tau_{1}$ and $\tau_{2}$ are the efolding times of the first and second infall episodes, respectively, and $t_{\max }$ indicates the delay of the beginning of the second infall. The coefficients $c_{1}$ and $c_{2}$ are obtained by imposing a fit to the currently observed surface mass densities of the thick- and thindisc components in the solar neighbourhood (obviously, $c_{2}$ is set to zero if $\left.t<t_{\max }\right)$. For the other models the formula gets simpler, 
Table 1. Input parameters for different models.

\begin{tabular}{lcccc}
\hline \hline Model & $\begin{array}{c}\text { e-folding time in infall law } \\
\left(\tau_{1}, \tau_{2} \text { or } \tau[\mathrm{Gyr}]\right)\end{array}$ & $\begin{array}{c}\text { Delay time for infall } \\
\left(t_{\text {max }}[\mathrm{Gyr}]\right)\end{array}$ & $\begin{array}{c}\text { Star formation efficiency } \\
\left(v \text { or } \tilde{v}\left[\mathrm{Gyr}^{-1}\right]\right)\end{array}$ & \begin{tabular}{c} 
gwIMF \\
$\mathrm{Canonical}^{a}$ \\
\hline Two-infall, thick disc (classical)
\end{tabular} \\
Two-infall, thin disc (classical) & 1.0 & - & 1.0 & $\mathrm{Canonical}^{a}$ \\
Two-infall, thick disc (revised) & 7.0 & 1.0 & 1.3 & $\mathrm{Canonical}^{a}$ \\
Two-infall, thin disc (revised) & 0.1 & - & 1.3 & $\mathrm{Cannonical}^{a}$ \\
Parallel, thick disc & 8.0 & 4.3 & 2.0 & $\mathrm{Canonical}^{a}$ \\
Parallel, thin disc & 0.5 & - & 1.0 & $\mathrm{Cannonical}^{a}$ \\
Galactic bulge & 7.0 & - & 25.0 & Salpeter $^{b}$ \\
Prototype elliptical galaxy & 0.1 & - & 1.8 & Top-heavy $^{c}$ \\
\hline
\end{tabular}

Notes. ${ }^{(a)}$ Kroupa (2002) IMF with $x=1.7$ in the high-mass domain $\left(x=1.35\right.$ for Salpeter IMF). ${ }^{(b)}$ Extrapolated Salpeter (1955) slope over the whole mass range. ${ }^{(c)}$ Slope flatter than Salpeter's, $x=1.1$, in the high-mass range (see Zhang et al. 2018, Romano et al. 2019).

$\frac{\mathrm{d} \mathscr{M}_{\text {inf }}(t)}{\mathrm{d} t}=c \mathrm{e}^{-t / \tau}$

where the different quantities have the usual meaning.

In all models, the star formation rate is implemented according to the Kennicutt-Schmidt law (Schmidt 1959, Kennicutt 1998). In the models for the Galactic bulge, halo and discs it reads

$\psi(r, t)=v\left[\frac{\sigma_{\mathrm{tot}}(r, t) \sigma_{\mathrm{gas}}(r, t)}{\sigma_{\mathrm{tot}}\left(r_{\odot}, t\right)^{2}}\right]^{(k-1)} \sigma_{\mathrm{gas}}^{k}(r, t)$,

where $\sigma_{\text {gas }}(r, t)$ is the surface gas density at a given radius and time, $\sigma_{\text {tot }}(r, t)$ is the total surface mass density at a given radius and time, $\sigma_{\text {tot }}\left(r_{\odot}, t\right)$ is the total surface mass density at the solar position, and $k=1.5$. In the one-zone model for ellipticals

$\psi(t)=\tilde{v} \mathscr{M}_{\mathrm{gas}}^{k}(t)$,

where $\mathscr{M}_{\text {gas }}(t)$ is the cold gas mass at time $t$ and $k=1$. Either way, the proportionality constants $(v, \tilde{v})$ are free parameters and vary from model to model.

As in previous works, the gwIMF is the canonical one (Kroupa 2002, with $x=1.7$ in the high-mass domain) for the solar neighbourhood models, while flatter gwIMFs are assumed in the models for spheroids.

Finally, galactic winds are considered only in the model for early-type galaxies.

The values of the main model parameters adopted in this work are summarized in Table 1

\subsection{Nucleosynthesis prescriptions}

The models presented in this paper account in detail for the finite stellar lifetimes, namely, the instantaneous recycling approximation is relaxed. However, they assume instantaneous, homogeneous mixing of the stellar ejecta within the ISM. As a consequence, these models can only predict average trends, while nothing can be said about the spread in the data.

As for the two-infall model, the adopted nucleosynthesis prescriptions are the same as model MWG-11 of Romano et al. (2019), namely, we adopt the yields by Ventura et al. (2013) for single low- and intermediate-mass stars and the yields by Limongi \& Chieffi (2018) for high-mass stars. In particular, in model MWG-11 the initial rotational velocities of massive stars are allowed to vary from the maximum value considered by Limongi \& Chieffi (2018), i.e., $300 \mathrm{~km} \mathrm{~s}^{-1}$, for $[\mathrm{Fe} / \mathrm{H}]<-1$ to zero at higher metallicities. Furthermore, the mass range for full collapse to black holes is set to $30-100 \mathrm{M}_{\odot}$ for $[\mathrm{Fe} / \mathrm{H}]<-1$, but is reduced to $60-100 \mathrm{M}_{\odot}$ for $[\mathrm{Fe} / \mathrm{H}] \geq-1$. This leads to a good agreement between model predictions and observations of CNO element abundances and isotopic ratios in the solar vicinity, as well as across the whole Galactic disc, in the framework of our model (see Romano et al. 2019).

All other models are run three times, one for each choice of nucleosynthetic yields corresponding to the assumptions of models MWG-05, MWG-06 and MWG-07 of Romano et al. (2019). In brief, we adopt the yields from Ventura et al. (2013) for (nonrotating) low- and intermediate-mass stars and the yields from Limongi \& Chieffi (2018, their recommended set R) with initial rotational velocities of 0 (models labeled ' $0 \mathrm{~km} / \mathrm{s}$ ' in Figs. 4 to 8), 150 (models labeled ' $150 \mathrm{~km} / \mathrm{s}$ ' in Figs. 4 to 8, or $300 \mathrm{~km} \mathrm{~s}$ '-1 (models labeled ' $300 \mathrm{~km} / \mathrm{s}$ ' in Figs. 4 to 8 ) for stars dying as core-collapse SNe. The interested reader is addressed to Romano et al. (2019, and references therein) for further details on the adopted stellar yield sets.

The nucleosynthetic outcome of SNeIa is also included in the models. In particular, we adopt the yields by Iwamoto et al. (1999) and the single-degenerate scenario for the progenitors following Matteucci \& Greggio (1986) and Matteucci \& Recchi (2001). The contribution to $\mathrm{C}, \mathrm{O}$ and $\mathrm{Mg}$ synthesis from SNeIa is negligible, while they produce huge amounts of Fe.

\section{Results}

\subsection{The solar vicinity}

\subsubsection{Two-infall model predictions}

In Fig. 3. left panel, we show [C/Fe] abundance ratios against $[\mathrm{Fe} / \mathrm{H}]$, on the right-hand side, we display the $[\mathrm{C} / \mathrm{O}]$ versus $[\mathrm{O} / \mathrm{H}]$ diagram. The circles refer to abundance determinations from high-resolution spectra of single dwarfs in the solar neighbourhood, after corrections for 3D and non-LTE effects (Amarsi et al. 2019). The solid lines are the predictions of the best fitting model by Romano et al. (2019), i.e., their model MWG-11. Theoretical uncertainties associated with changes in the adopted stellar yields are highlighted (shaded areas); though they are large, the direct comparison with a good data set provides a sensible way to discriminate among various nucleosynthesis prescriptions. The dashed lines refer to the predictions of model MWG11 after introducing the improvements to the two-infall evolutionary scheme recently suggested by Spitoni et al. (2019). 

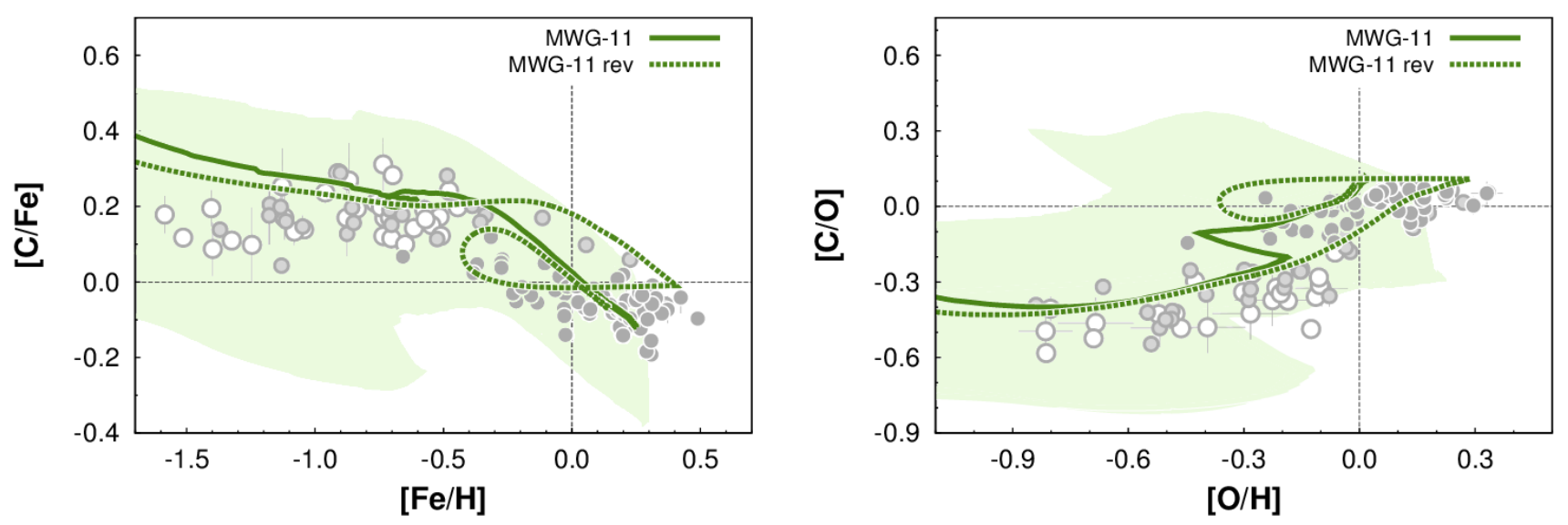

Fig. 3. Carbon-to-iron (left panel) and carbon-to-oxygen (right panel) abundance ratios as functions of $[\mathrm{Fe} / \mathrm{H}]$ and $[\mathrm{O} / \mathrm{H}]$, respectively, in the solar vicinity. The solid lines show the predictions of model MWG-11 by Romano et al. (2019); theoretical uncertainties arising from stellar nucleosynthesis are highlighted by pale green areas. The dashed lines show the predictions of model MWG-11 revised following Spitoni et al. (2019 see text and Table 1). The symbols represent 3D non-LTE abundance estimates from high-resolution spectra of thin-disc, thick-disc and high$\alpha$ halo stars (dark grey filled circles, light grey filled circles and empty circles, respectively; Amarsi et al. 2019); in most cases, the observational error lies within the symbol size. All abundance ratios are normalised to the solar photospheric composition by|Asplund et al. (2009).

In the metallicity range $-1.4<[\mathrm{Fe} / \mathrm{H}]<-0.4(-0.9<$ $[\mathrm{O} / \mathrm{H}]<0.0)$, objects classified as high- $\alpha$ halo stars ${ }^{3}$ or thickdisc stars overlap each other. At variance with halo stars, some thick-disc members are found at higher metallicities, up to $[\mathrm{Fe} / \mathrm{H}] \sim 0.2$, or $[\mathrm{O} / \mathrm{H}] \sim 0.3$ (see also Bensby et al. 2007; Adibekyan et al. 2011). In its classical formulation, the twoinfall model cannot accommodate thick-disc stars with $[\mathrm{Fe} / \mathrm{H}]>$ $-0.6($ or $[\mathrm{O} / \mathrm{H}]>-0.2)$. However, when a longer duration is assumed for the inner-halo/thick-disc phase, as suggested by precise stellar ages provided by asteroseismology (Spitoni et al. 2019, and references therein), stars as metal-rich as $[\mathrm{Fe} / \mathrm{H}] \simeq 0.4$ (or $[\mathrm{O} / \mathrm{H}] \simeq 0.3$ ) are predicted to form in the thick-disc component, in full agreement with the observations. As shown in Fig. 3 . the theoretical $[\mathrm{C} / \mathrm{Fe}]$ and $[\mathrm{C} / \mathrm{O}]$ ratios of these stars agree well (within $0.06 \mathrm{dex}$ ) with the observed ratios. The $[\mathrm{C} / \mathrm{Fe}]$ and $[\mathrm{C} / \mathrm{O}]$ ratios of the whole sample, though, appear to be slightly overestimated.

Overall, the revised MWG-11 model reproduces the bunch of local data satisfactorily well, especially if we consider that a sizeable fraction of the most metal-rich stars in the solar neighbourhood (i.e., those with $[\mathrm{Fe} / \mathrm{H}]>0.25 \mathrm{dex}$ ) might actually be not born locally, but migrated from other radii (e.g., Minchev et al. 2013; Kubryk et al. 2015, see also Schönrich \& Binney 2009), and would, thus, reflect different evolutionary paths. Indeed, their old ages (e.g., Anders et al. 2017, and references therein) fully support this hypothesis.

\subsubsection{Parallel model predictions}

In the previous section, we discuss the results of model MWG11 , where an abrupt transition is supposed to occur between a regime that favours the formation of massive fast rotators at low metallicities and a regime that leads mostly to slowly- or non-rotating stars above $[\mathrm{Fe} / \mathrm{H}]=-1.0$ (Romano et al. 2019). These, or similar, assumptions allow to obtain a satisfactory fit to the abundance distribution of most chemical elements in the Galaxy (Romano et al.2019, see also Prantzos et al.2018). How-

\footnotetext{
3 These are possibly stars formed in situ, as opposed to low- $\alpha$ halo stars that have likely been accreted from disrupting dwarf satellites (see Nissen et al. 2014, and references therein).
}

ever, the dependence of stellar rotation on metallicity is basically unknown, and other factors might act as the prime drivers behind the variations in the angular momenta of the proto-star clouds. Therefore, in the following we discuss separately, for each Galactic component, the results of three models that assume different fixed values for the initial rotational velocity of all massive stars.

In Fig. 4 we show the predictions of the parallel model by Grisoni et al. (2017) obtained when three different sets of stellar yields, corresponding to models MWG-05, MWG-06 and MWG-07 of Romano et al. (2019), are implemented.

For stars in the mass range $1-8 \mathrm{M}_{\odot}$, the yields are from Ventura et al. (2013, and private communication) and are computed for six different values of the initial metallicity of the stars, namely, $Z=0.0003,0.001,0.004,0.008,0.018$, and 0.04; notably, this includes a set computed for super-solar metallicity stars. The yields for massive stars are from Limongi \& Chieffi (2018). In this case, the modelled stars have initial metallicities of $[\mathrm{Fe} / \mathrm{H}]=-3,-2,-1$, and 0 dex, corresponding to $Z=3.236 \times 10^{-5}, 3.236 \times 10^{-4}, 3.236 \times 10^{-3}$, and $1.345 \times 10^{-2}$; unfortunately, no models are available at super-solar metallicities. While the stellar models of Ventura et al. (2013) do not include stellar rotation, Limongi \& Chieffi (2018) provide yields for non-rotating stars, as well as yields for fast rotators. In Fig. 4 we indicate with the labels ' $300 \mathrm{~km} / \mathrm{s}$ ', ' $150 \mathrm{~km} / \mathrm{s}$ ' and ' $0 \mathrm{~km} / \mathrm{s}$ ' models in which all massive stars rotate with $v_{\text {rot }}=300$ or $150 \mathrm{~km} \mathrm{~s}^{-1}$, or do not rotate at all, respectively.

In the parallel model, the formation of the thick and thin discs are completely disentangled. The thick-disc model implementing the yields for non-rotating massive stars (light blue lines in Fig. (4) agrees very well with the sparse data for metal-rich thick-disc stars in the $[\mathrm{C} / \mathrm{Fe}]$ versus $[\mathrm{Fe} / \mathrm{H}]$ plane, it marginally agrees with the points at $[\mathrm{Fe} / \mathrm{H}]<-0.2$ in the same diagram, but it fails by far to reproduce the $[\mathrm{C} / \mathrm{O}]$ ratios over the full $[\mathrm{O} / \mathrm{H}]$ range. When rotation is considered (blue and dark blue lines in Fig. 47, a good agreement is found between model predictions and observations in the $[\mathrm{C} / \mathrm{O}]$ versus $[\mathrm{O} / \mathrm{H}]$ plane, but the agreement with the observed $[\mathrm{C} / \mathrm{Fe}]$ ratios for $[\mathrm{Fe} / \mathrm{H}]>-0.2$ sensibly worsens. The transition from a regime favouring fast rotators to a regime where fast rotators are strongly suppressed when moving from low to high metallicities offers, in principle, an elegant 

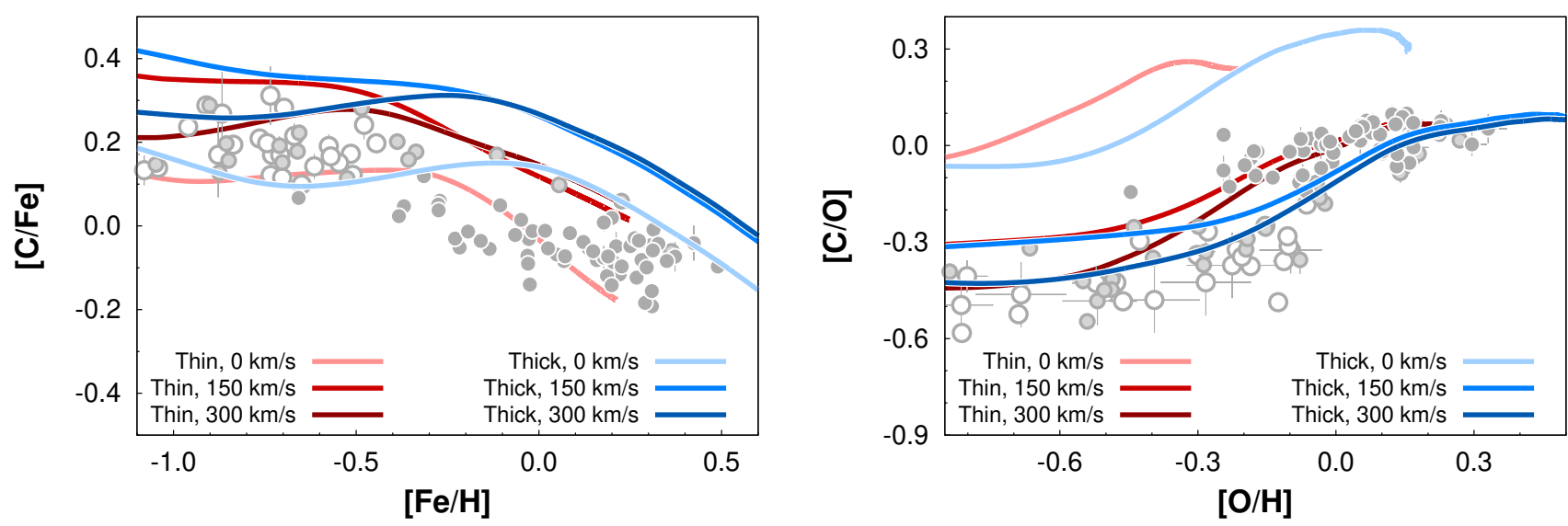

Fig. 4. Similar to the previous figure. Now we show the predictions of the parallel model for thick- and thin-disc formation by Grisoni et al. (2017), in which we include up-to-date stellar nucleosynthetic yields (see text and Romano et al. 2019).
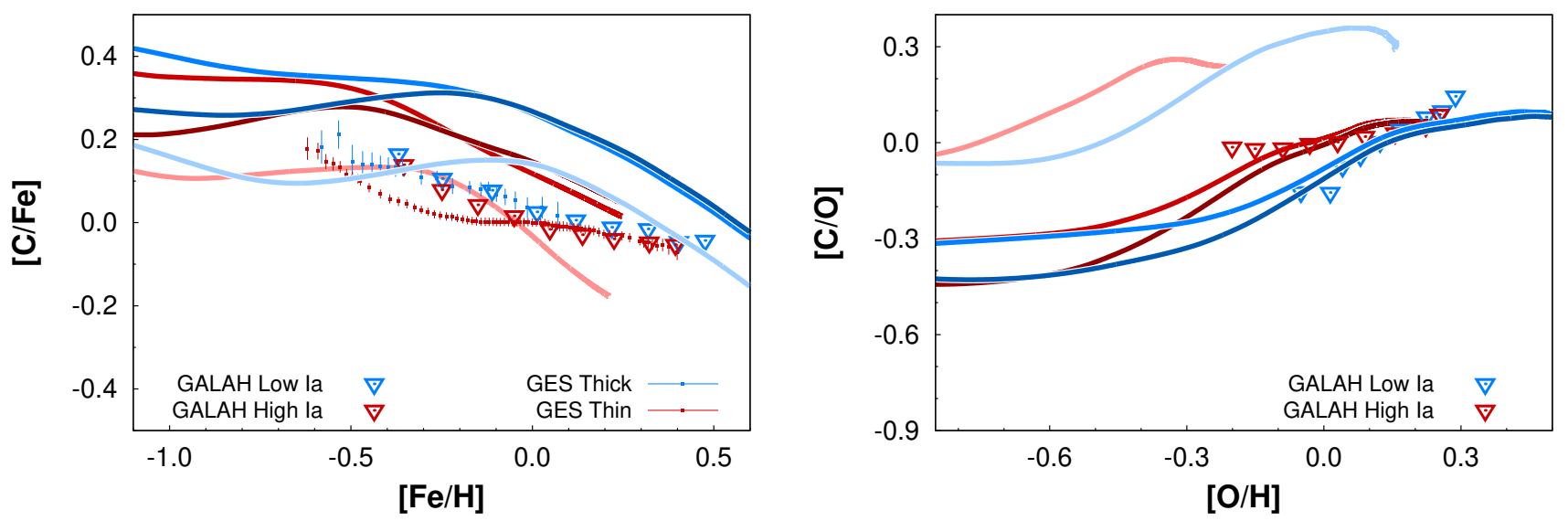

Fig. 5. Same as Fig. 4. but now the model predictions are compared to the observed trends inferred for: (i) thin- and thick-disc dwarf stars from GES iDR5 (red and blue dots, respectively; Franchini et al. 2020); (ii) high-Ia and low-Ia GALAH DR2 stars (red and blue upside-down triangles, respectively; Griffith et al.2019). Zero-point shifts are applied to the data (see Sect. 2.1].

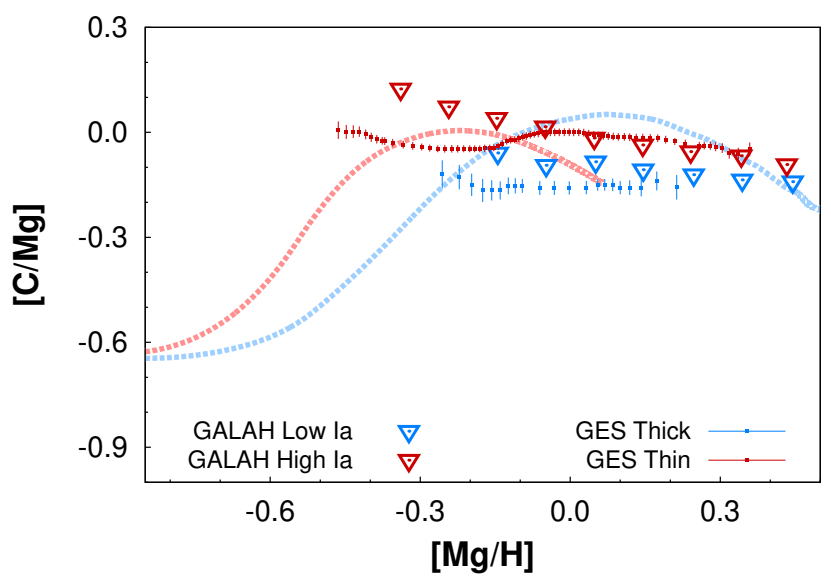

Fig. 6. $[\mathrm{C} / \mathrm{Mg}]-[\mathrm{Mg} / \mathrm{H}]$ diagram. Data are from GES iDR5 and GALAH DR2 (dots and upside-down triangles, respectively) and include zero-point shifts (see text). Predicted trends for the thick (blue line) and thin (red line) discs are from the parallel model (Grisoni et al. 2017) with nucleosynthesis prescriptions as model MWG-01 of Romano et al. (2019).

solution to this problem (see Fig. 3 and Romano et al. 2019), but in order to test this hypothesis properly, a denser grid of massive star yields, comprising results obtained for intermediate values of the initial rotational velocities, is definitely needed. The models for the thin disc (Fig. 4, bundles of lines in shades of red) strengthen the above conclusions.

In principle, nowadays large modern spectroscopic surveys allow us to establish tight abundance trends through the analysis of thousands of stars (see Sect. 2.1). In Fig. 5. we compare the same theoretical predictions displayed in Fig. 4 to the low-Ia and high-Ia sequences constructed by Griffith et al. (2019) using GALAH DR2 data (upside-down triangles), as well as to the binned running averages for the thick- and thin-disc components from GES iDR5 data (blue and red dots, respectively, the vertical bars represent the standard deviations; Franchini et al. 2020).

In the $[\mathrm{C} / \mathrm{O}]-[\mathrm{O} / \mathrm{H}]$ plane, a strikingly good agreement is found between the low-Ia/high-Ia sequences of Griffith et al. (2019) and the predictions of the thick-disc/thin-disc models featuring rotating massive stars, with the exception of the highmetallicity ends of the tracks, where the models do not match the sudden increase in $[\mathrm{C} / \mathrm{O}]$ displayed by the observations (Fig. 5 , right panel). Franchini et al. (2020) do not provide oxygen abundances for their sample stars, therefore, we cannot compare our theoretical trends to GES data in a $[\mathrm{C} / \mathrm{O}]$ versus $[\mathrm{O} / \mathrm{H}]$ diagram. When iron is used as a metallicity tracer (Fig. 5, left panel), the agreement with the data is much weaker. Although systematic offsets may be present, it is clear that the models com- 

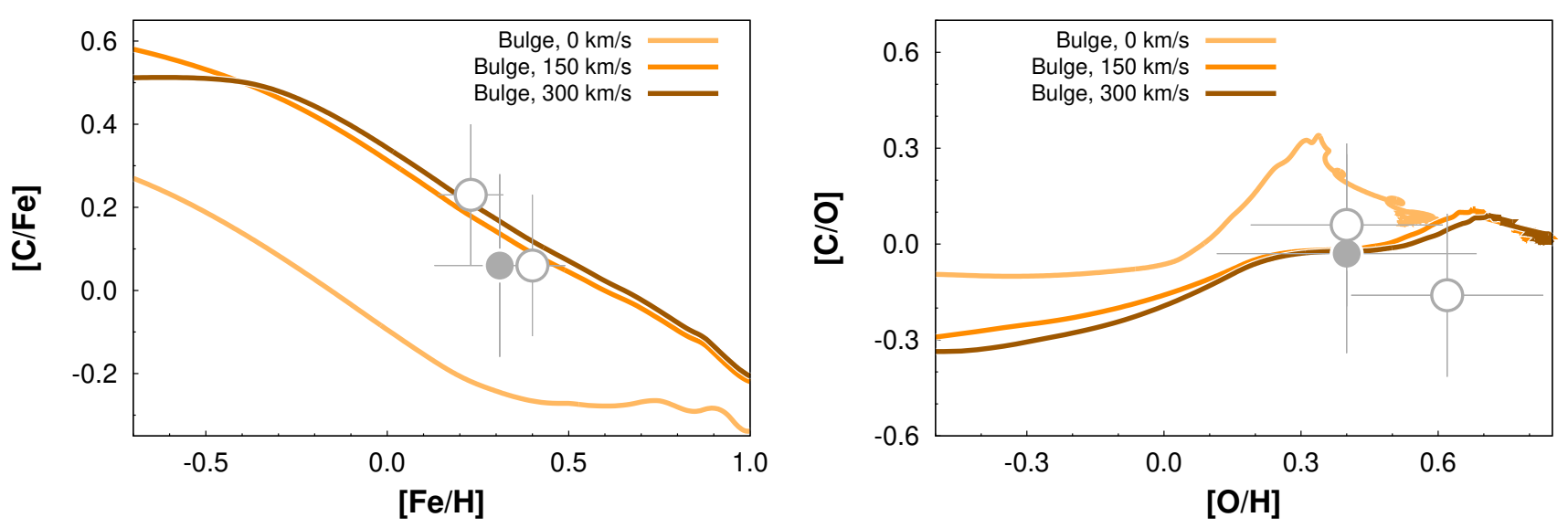

Fig. 7. Carbon-to-iron (left panel) and carbon-to-oxygen (right panel) abundance ratios as functions of $[\mathrm{Fe} / \mathrm{H}]$ and $[\mathrm{O} / \mathrm{H}]$, respectively, in the Galactic bulge. The solid lines show the predictions of the chemical evolution model for the bulge by Matteucci et al. (2019) including the same nucleosynthesis prescriptions as models MWG-05 (brown), MWG-06 (orange), and MWG-07 (yellow) of Romano et al. (2019). Data for microlensed bulge dwarfs from Johnson et al. (2008 filled circles) and Cohen et al. (2009 empty circles) are also shown. All abundance ratios are normalised to the solar photospheric abundances by Asplund et al. (2009).

pletely fail to reproduce the flattening of $[\mathrm{C} / \mathrm{Fe}]$ versus $[\mathrm{Fe} / \mathrm{H}]$ for $[\mathrm{Fe} / \mathrm{H}]>-0.2-0.0$. This matches the absence of an increase in the theoretical curves for $[\mathrm{C} / \mathrm{O}]$ versus $[\mathrm{O} / \mathrm{H}]$ at the highmetallicity end and might be due to underestimated $\mathrm{C}$ yields from either low-mass or massive stars, or both. In particular, keeping using solar-metallicity yields for massive stars when the metallicity exceeds solar could lead to underestimate C pollution from Wolf-Rayet stars (see Chiosi \& Maeder 1986; Maeder 1992 , for a discussion of the effects of metallicity on $C$ production from massive stars).

This section wraps up with a couple of comments on the evolution of the $[\mathrm{C} / \mathrm{Mg}]$ ratio as a function of $[\mathrm{Mg} / \mathrm{H}]$. Magnesium is relatively easy to observe in stars and, thus, it is often used in place of oxygen as a typical $\alpha$-element or metallicity tracer. However, its origin is far less understood than that of oxygen. As a matter of fact, GCE models generally fail to reproduce Mg evolution (see Romano et al. 2010, Prantzos et al. 2018, and references therein) unless some ad hoc corrections are made to the yields (e.g., François et al. 2004). The yields presented by Kobayashi et al. (2006) and Nomoto et al. (2013) constitute a notable exception, in that they provide a satisfactory fit to the data in the $[\mathrm{Mg} / \mathrm{Fe}]-[\mathrm{Fe} / \mathrm{H}]$ plane without any need for adjustments (see, e.g., Fig. 10 of Nomoto et al.2013).

In Fig. 6 we display the predictions of the parallel model implementing $\mathrm{C}$ and $\mathrm{Mg}$ yields for massive stars by Nomoto et al. (2013). The low-Ia/high-Ia sequences by Griffith et al. (2019) are fitted only marginally, while the almost flat trends of Franchini et al. (2020) remain unexplained. We conclude that any meaningful comparison between our GCE model predictions and observations should better involve oxygen rather than magnesium. For this reason, in the remainder of this paper we comment only on $\mathrm{C}, \mathrm{O}$, and $\mathrm{Fe}$ abundance determinations.

\subsection{The Galactic bulge}

It has been pointed out elsewhere (Fulbright et al. 2007; Cescutti et al. 2009) that $\mathrm{C}$ abundance measurements in the Galactic bulge are extremely important in that they provide a test for the predicted increase in the carbon yield due to enhanced mass loss through stellar winds from metal-rich massive stars.
Actually, now a non-negligible number of metal-rich stars is found in the solar neighbourhood, which provides a sensible testbed for this idea (see Sect. 4.1 and Figs. 3 to 5 . Adding information from bulge stars, however, permits to study not only the dependence on stellar metallicity, but also that on the environment. In fact, by comparing $\mathrm{C}$ abundances measured in dwarf stars with similarly high metal content that belong to either the bulge or the local disc, we can contrast the properties of metalrich stars that formed either fast in a spheroid at high redshift, or at a slower pace and more recently in a flattened structure.

With $[\mathrm{O} / \mathrm{H}]=0.4 \pm 0.28,0.4 \pm 0.21$, and $0.62 \pm 0.21$, the three microlensed bulge dwarfs observed at high resolution by Johnson et al. (2008) and Cohen et al. (2009) probe the highmetallicity tail of the metallicity distribution function of bulge stars. The $[\mathrm{C} / \mathrm{Fe}]$ ratios determined for these stars lie in the range $\sim 0.0-0.3$, while the $[\mathrm{C} / \mathrm{O}]$ ratios are consistent with solar. Chemical evolution models for the bulge (Matteucci et al. 2019) that adopt the same nucleosynthesis prescriptions as models MWG05 and MWG-06 of Romano et al. (2019), namely, the yields by Ventura et al. (2013) for low- and intermediate-mass stars and those by Limongi \& Chieffi (2018) for fast-rotating massive stars (with $v_{\text {rot }}=300$ and $150 \mathrm{~km} \mathrm{~s}^{-1}$, respectively), match very well the $[\mathrm{C} / \mathrm{Fe}]$ and $[\mathrm{C} / \mathrm{O}]$ bulge data; on the contrary, the adoption of non-rotating stellar yields leads to severely underestimate (overestimate) the $[\mathrm{C} / \mathrm{Fe}]([\mathrm{C} / \mathrm{O}])$ ratio (see Fig. 7]).

More $\mathrm{C}$ abundances of dwarf stars, free from stellar evolutionary effects, in the bulge are urgently needed before we can draw any firm conclusion about carbon evolution in the central regions of the Galaxy.

\subsection{Massive elliptical galaxies}

In Fig. 8, we display the evolution of the mean carbon-to-oxygen abundance ratio as a function of $[\mathrm{O} / \mathrm{H}]$ in the stellar population of the prototype massive elliptical galaxy (thick solid lines, different shades of purple refer to different nucleosynthesis prescriptions, corresponding to different initial rotational velocities of the core-collapse SN progenitors). The galaxy is assumed to form stars for about 1 Gyr through a sequence of starbursts, lasting $50 \mathrm{Myr}$ each, alternating with similarly long quiescent pe- 


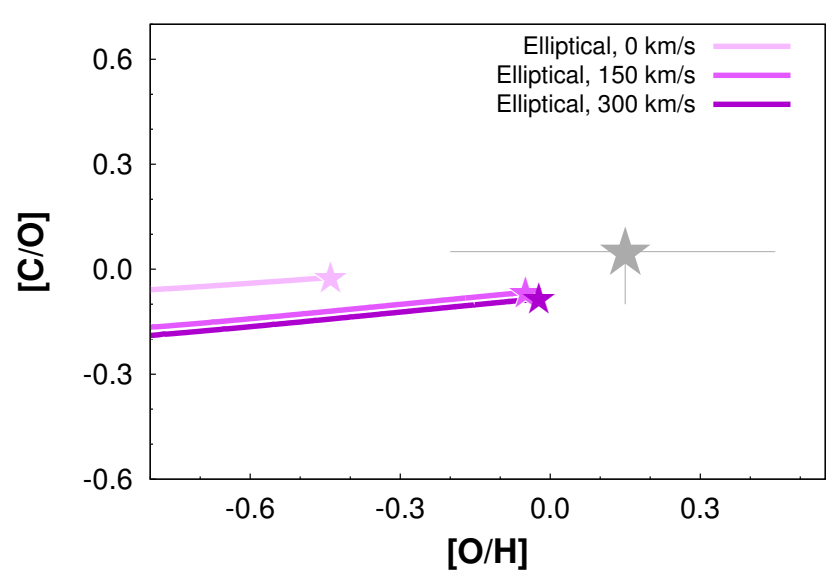

Fig. 8. Average carbon-to-oxygen abundance ratio as a function of $[\mathrm{O} / \mathrm{H}]$ in the stellar population of the prototype massive elliptical galaxy. The thick solid lines show the evolution of the mass-averaged abundances of the stellar population. The starlets mark the ending points of the theoretical tracks. Different shades of purple refer to different nucleosynthesis prescriptions (see text). The big grey star indicates the values of the ratios estimated from absorption line indices of local galaxies ( $\mathrm{JO}-$ hansson et al. 2012).

riods 4 (see Romano et al. 2019). The evolutionary tracks end at the time in which a powerful outflow stops the star formation, that is, when the system has reached a stellar mass of about $2 \times 10^{11} \mathrm{M}_{\odot}$. With our choice of the star formation history, the typical massive early-type galaxy is seen to form stars at rates of several hundreds of solar masses per year at redshifts 2 to 3 , in agreement with observations of submillimeter galaxies (e.g., Ivison et al. 2011).

The big grey star with generous error bars indicates the region of the $[\mathrm{C} / \mathrm{O}]-[\mathrm{O} / \mathrm{H}]$ diagram where passively-evolving, massive SDSS early-type galaxies are found (Johansson et al. 2012). Observational estimates of the abundance ratios rely on index measurements of unresolved stellar populations. In Fig. 8 we display mean theoretical stellar abundances averaged on the stellar mass. A more precise comparison should involve abundances averaged on the visual light. However, for massive galaxies formed at high redshifts the latter do not differ significantly from the mass-averaged ones (see, e.g., Romano et al. 2002; Matteucci 2012, and references therein). The ending points of the tracks, i.e., the quantities that have to be compared with the observations, are highlighted by the starlets. It is seen that models in which the majority of massive stars are rotating fast comply better with the observations.

It is worth emphasising at this point that our models for elliptical galaxies adopt a top-heavy gwIMF (see Sect. 3.1. The adoption of a canonical gwIMF would lead to significantly lower mean stellar metallicities $([\mathrm{O} / \mathrm{H}] \simeq-0.7$ for the models with fast rotators). Thus, the adoption of a gwIMF biased towards massive stars appears to be a fundamental assumption in order to reproduce the data.

\section{Discussion}

While important uncertainties still plague stellar evolution and nucleosynthesis studies, the flood of data secured by large modern spectroscopic surveys makes it possible, in principle, to de-

\footnotetext{
4 The results do not change if a continuous star formation history of similar duration is assumed instead.
}

fine tight abundance trends that can be used to constrain sensibly both stellar and galactic chemical evolution models. In practice, poorly-understood, possibly significant systematics currently hamper our ability to derive accurate trends of abundance ratios over the full metallicity range, even for local samples.

We have shown that the observational scatter of individual points in the $[\mathrm{C} / \mathrm{Fe}]$ versus $[\mathrm{Fe} / \mathrm{H}]$ and $[\mathrm{C} / \mathrm{Mg}]$ versus $[\mathrm{Mg} / \mathrm{H}]$ diagrams and the differences in the trends found by the two large surveys considered in this study (GES and GALAH) make it challenging the comparison of the observations with the predictions of GCE models. While individual scattered data points can be fitted reasonably well, the comparison between mean observed and theoretical trends gives less sound results. However, if the scatters are intrinsic, as suggested in Sect. 2.1, the observational trends should be taken with caution, since they may be affected by biases depending on the nature, origin, and number of individual points used to build them. Moreover, in such a case the usage of mean trends may mask the origin and sources of the scatter. Yet, GCE models, by construction, predict the climate, not the weather (they only account for large-scale phenomena); much more complex (and computationally expensive) hydrodynamical simulations are needed to address specifically the scatter in the data (see, e.g., Emerick et al. 2019), but this is beyond the scope of the present paper.

Regarding the $[\mathrm{C} / \mathrm{O}]-[\mathrm{O} / \mathrm{H}]$ diagram for local disc stars, a strikingly good agreement is found between the predictions of our GCE models and targeted observations of thick- and thindisc stars, as well as the average trends derived from the analysis of GALAH DR2 data (Griffith et al.2019).

Overall, our GCE models reproduce satisfactorily well the high resolution $\mathrm{C}, \mathrm{O}$, and $\mathrm{Fe}$ data for solar neighbourhood stars under the assumption that massive stars rotate fast at low metallicities, while they rotate much slowly, or not at all, for $[\mathrm{Fe} / \mathrm{H}]>$ -1.0 . The bulge and elliptical galaxy data are better matched by models with only fast rotators. This hints to a dependence of stellar rotation on environmental factors, such as pressure, temperature and density of the ambient medium, and/or the effects of the permeating radiation field (see also Romano et al. 2019).

In the framework of our models, more than 60 per cent of the solar $\mathrm{C}$ abundance comes from massive stars. This percentage increases, becoming $\sim 70$ per cent, for stars in spheroids. Therefore, according to our calculations the majority of $\mathrm{C}$ in the Universe comes from massive (fast) rotators, with a non-negligible contribution from intermediate-mass stars. In the Galactic bulge and massive elliptical galaxies $\mathrm{C}$ production from high-mass stars is boosted by the adoption of gwIMFs flatter than the canonical one in the high-mass domain. In general, top-heavy gwIMFs are required to explain other observed properties of spheroids (see Matteucci 2012) and naturally emerge from the IGIMF theory (Jeřábková et al. 2018; Yan et al. 2019, and references therein).

Finally, it is worth emphasising that, unlike other studies in the literature, we have not applied ad hoc corrections to the adopted stellar yield tables to make the model predictions match the observed trends. Yet, we are able to obtain a satisfactory agreement between our model predictions and the observations (when taking into account all the possible sources of uncertainties). That is very encouraging, as it means that $\mathrm{C}$ production in stars is now quantitatively pretty well understood.

\section{Conclusions}

In this paper, we present GCE model results for different Galactic components, namely the Galactic thick and thin discs and the 
bulge, as well for passively-evolving, massive early-type galaxies. In particular, we deal with the evolution of carbon, as manifested in the $[\mathrm{C} / \mathrm{Fe}]$ versus $[\mathrm{Fe} / \mathrm{H}]$ and $[\mathrm{C} / \mathrm{O}]$ versus $[\mathrm{O} / \mathrm{H}]$ planes ( $\mathrm{Mg}$ is a less reliable metallicity tracer from the theoretician point of view).

We conclude that:

1. GCE models fit reasonably well individual data points from targeted high-resolution studies of nearby unevolved stars that belong to the inner halo, thick- and thin-disc components. In particular, both the revised two-infall model recently proposed by Spitoni et al. (2019) and the parallel model by Grisoni et al. (2017) fit very nicely the data in the $[\mathrm{C} / \mathrm{O}]-[\mathrm{O} / \mathrm{H}]$ diagram.

2. While the models fit very well the $[\mathrm{C} / \mathrm{O}]$ versus $[\mathrm{O} / \mathrm{H}]$ trends derived from GALAH DR2 data, several problems are apparent when trying to match the GES and GALAH trends in the $[\mathrm{C} / \mathrm{Fe}]-[\mathrm{Fe} / \mathrm{H}]$ and $[\mathrm{C} / \mathrm{Mg}]-[\mathrm{Mg} / \mathrm{H}]$ planes. This is partly due to the more uncertain yields of Fe. However, it must also be kept in mind that possibly significant systematics might hamper our ability to derive accurate trends of abundance ratios, even for local stars.

3. The use of $\mathrm{Mg}$ as a metallicity tracer leads to the worst match between theoretical predictions and data, partly due to the poorly-known nucleosynthesis of $\mathrm{Mg}$ in stars.

4. The assumption that most massive stars rotate fast during the earliest phases of galactic evolution leads to the best agreement with the observations for both the Galactic discs, bulge and massive ellipticals.

5. In order to reproduce the observations, it seems unavoidable that the gwIMF in spheroids is biased towards massive stars. The flattest slopes are expected in the most massive ellipticals. This conclusion strengthens previous claims about the need for a top-heavy gwIMF in submillimeter galaxies based on measurements of ${ }^{13} \mathrm{C} /{ }^{18} \mathrm{O}$ isotopic ratios in the gas phase (Zhang et al. 2018).

Acknowledgements. This work benefited from the International Space Science Institute (ISSI) in Bern, $\mathrm{CH}$, and the International Space Science InstituteBeijing (ISSI-BJ) in Beijing, $\mathrm{CN}$, thanks to the funding of the team "Chemical abundances in the ISM: the litmus test of stellar IMF variations in galaxies across cosmic time" (PIs D. Romano, Z.-Y. Zhang).

\section{References}

Adibekyan, V. Z., Santos, N. C., Sousa, S. G., \& Israelian, G. 2011, A\&A, 535, L11

Amarsi, A. M., Nissen, P. E., \& Skúladóttir, Á. 2019, A\&A, 630, A104

Anders, F., Chiappini, C., Minchev, I., et al. 2017, A\&A, 600, A70

Asplund, M., Grevesse, N., Sauval, A. J., \& Scott, P. 2009, ARA\&A, 47, 481

Bastian, N. \& Lardo, C. 2018, ARA\&A, 56, 83

Bensby, T. \& Feltzing, S. 2006, MNRAS, 367, 1181

Bensby, T., Feltzing, S., Gould, A., et al. 2017, A\&A, 605, A89

Bensby, T., Feltzing, S., Johnson, J. A., et al. 2010, A\&A, 512, A41

Bensby, T., Feltzing, S., \& Oey, M. S. 2014, A\&A, 562, A71

Bensby, T., Zenn, A. R., Oey, M. S., \& Feltzing, S. 2007, ApJ, 663, L13

Bertran de Lis, S., Allende Prieto, C., Majewski, S. R., et al. 2016, A\&A, 590, A74

Bethe, H. A. 1939, Physical Review, 55, 434

Buder, S., Lind, K., Ness, M. K., et al. 2019, A\&A, 624, A19

Burbidge, E. M., Burbidge, G. R., Fowler, W. A., \& Hoyle, F. 1957, Reviews of Modern Physics, 29, 547

Cannon, R. D., Croke, B. F. W., Bell, R. A., Hesser, J. E., \& Stathakis, R. A. 1998, MNRAS, 298, 601

Carilli, C. L. \& Walter, F. 2013, ARA\&A, 51, 105

Cescutti, G., Matteucci, F., McWilliam, A., \& Chiappini, C. 2009, A\&A, 505, 605

Cescutti, G., Romano, D., Matteucci, F., Chiappini, C., \& Hirschi, R. 2015, A\&A, 577, A139
Charbonnel, C. 1994, A\&A, 282, 811

Chiappini, C., Matteucci, F., \& Gratton, R. 1997, ApJ, 477, 765

Chiappini, C., Matteucci, F., \& Romano, D. 2001, ApJ, 554, 1044

Chiosi, C. \& Maeder, A. 1986, ARA\&A, 24, 329

Cicone, C., Maiolino, R., Sturm, E., et al. 2014, A\&A, 562, A21

Cohen, J. G., Thompson, I. B., Sumi, T., et al. 2009, ApJ, 699, 66

Conroy, C., Graves, G. J., \& van Dokkum, P. G. 2014, ApJ, 780, 33

De Silva, G. M., Freeman, K. C., Bland-Hawthorn, J., et al. 2015, MNRAS, 449 , 2604

Emerick, A., Bryan, G. L., \& Mac Low, M.-M. 2019, arXiv e-prints, arXiv: 1909.04695

Feruglio, C., Maiolino, R., Piconcelli, E., et al. 2010, A\&A, 518, L155

François, P., Matteucci, F., Cayrel, R., et al. 2004, A\&A, 421, 613

Franchini, M., Morossi, C., Di Marcantonio, P., et al. 2020, ApJ, 888, 55

Fulbright, J. P., McWilliam, A., \& Rich, R. M. 2007, ApJ, 661, 1152

Gilmore, G., Randich, S., Asplund, M., et al. 2012, The Messenger, 147, 25

Gratton, R., Bragaglia, A., Carretta, E., et al. 2019, A\&A Rev., 27, 8

Gratton, R. G., Sneden, C., Carretta, E., \& Bragaglia, A. 2000, A\&A, 354, 169

Griffith, E., Johnson, J. A., \& Weinberg, D. H. 2019, ApJ, 886, 84

Grisoni, V., Cescutti, G., Matteucci, F., et al. 2020, MNRAS, 492, 2828

Grisoni, V., Matteucci, F., Romano, D., \& Fu, X. 2019, MNRAS, 489, 3539

Grisoni, V., Spitoni, E., \& Matteucci, F. 2018, MNRAS, 481, 2570

Grisoni, V., Spitoni, E., Matteucci, F., et al. 2017, MNRAS, 472, 3637

Harbeck, D., Smith, G. H., \& Grebel, E. K. 2003, AJ, 125, 197

Ivison, R. J., Papadopoulos, P. P., Smail, I., et al. 2011, MNRAS, 412, 1913

Iwamoto, K., Brachwitz, F., Nomoto, K., et al. 1999, ApJS, 125, 439

Jeřábková, T., Hasani Zonoozi, A., Kroupa, P., et al. 2018, A\&A, 620, A39

Johansson, J., Thomas, D., \& Maraston, C. 2012, MNRAS, 421, 1908

Johnson, J. A., Gaudi, B. S., Sumi, T., Bond, I. A., \& Gould, A. 2008, ApJ, 685, 508

Kennicutt, Jr., R. C. 1998, ApJ, 498, 541

Kobayashi, C., Umeda, H., Nomoto, K., Tominaga, N., \& Ohkubo, T. 2006, ApJ, 653,1145

Kroupa, P. 2002, in Astronomical Society of the Pacific Conference Series, Vol. 285, Modes of Star Formation and the Origin of Field Populations, ed. E. K. Grebel \& W. Brandner, 86

Kubryk, M., Prantzos, N., \& Athanassoula, E. 2015, A\&A, 580, A126

Lagarde, N., Reylé, C., Robin, A. C., et al. 2019, A\&A, 621, A24

Limongi, M. \& Chieffi, A. 2018, ApJS, 237, 13

Maas, Z. G., Gerber, J. M., Deibel, A., \& Pilachowski, C. A. 2019, ApJ, 878, 43

Maeder, A. 1992, A\&A, 264, 105

Majewski, S. R., Schiavon, R. P., Frinchaboy, P. M., et al. 2017, AJ, 154, 94

Marigo, P. 2002, A\&A, 387, 507

Matteucci, F. 2012, Chemical Evolution of Galaxies (Berlin Heidelberg: Springer-Verlag)

Matteucci, F. \& Brocato, E. 1990, ApJ, 365, 539

Matteucci, F. \& François, P. 1989, MNRAS, 239, 885

Matteucci, F. \& Greggio, L. 1986, A\&A, 154, 279

Matteucci, F., Grisoni, V., Spitoni, E., et al. 2019, MNRAS, 487, 5363

Matteucci, F. \& Recchi, S. 2001, ApJ, 558, 351

Matteucci, F., Romano, D., Arcones, A., Korobkin, O., \& Rosswog, S. 2014, MNRAS, 438, 2177

Matteucci, F., Romano, D., \& Molaro, P. 1999, A\&A, 341, 458

Minchev, I., Chiappini, C., \& Martig, M. 2013, A\&A, 558, A9

Nissen, P. E., Chen, Y. Q., Carigi, L., Schuster, W. J., \& Zhao, G. 2014, A\&A, 568, A25

Nissen, P. E. \& Schuster, W. J. 2010, A\&A, 511, L10

Nomoto, K., Kobayashi, C., \& Tominaga, N. 2013, ARA\&A, 51, 457

Prantzos, N., Abia, C., Limongi, M., Chieffi, A., \& Cristallo, S. 2018, MNRAS, 476,3432

Romano, D., Karakas, A. I., Tosi, M., \& Matteucci, F. 2010, A\&A, 522, A32

Romano, D., Matteucci, F., Salucci, P., \& Chiappini, C. 2000, ApJ, 539, 235

Romano, D., Matteucci, F., Zhang, Z.-Y., Ivison, R. J., \& Ventura, P. 2019, MNRAS, 490, 2838

Romano, D., Matteucci, F., Zhang, Z. Y., Papadopoulos, P. P., \& Ivison, R. J. 2017, MNRAS, 470, 401

Romano, D., Silva, L., Matteucci, F., \& Danese, L. 2002, MNRAS, 334, 444

Romano, D., Tosi, M., Matteucci, F., \& Chiappini, C. 2003, MNRAS, 346, 295

Salpeter, E. E. 1955, ApJ, 121, 161

Schmidt, M. 1959, ApJ, 129, 243

Schönrich, R. \& Binney, J. 2009, MNRAS, 396, 203

Spitoni, E., Romano, D., Matteucci, F., \& Ciotti, L. 2015, ApJ, 802, 129

Spitoni, E., Silva Aguirre, V., Matteucci, F., Calura, F., \& Grisoni, V. 2019, A\&A, 623, A60

Tinsley, B. M. 1980, Fund. Cosmic Phys., 5, 287

Ventura, P., Di Criscienzo, M., Carini, R., \& D'Antona, F. 2013, MNRAS, 431, 3642

Vidal-Madjar, A., Désert, J. M., Lecavelier des Etangs, A., et al. 2004, ApJ, 604, L69

Yan, Z., Jerabkova, T., Kroupa, P., \& Vazdekis, A. 2019, A\&A, 629, A93

York, D. G., Adelman, J., Anderson, J. E. J., et al. 2000, AJ, 120, 1579

Zhang, Z.-Y., Romano, D., Ivison, R. J., Papadopoulos, P. P., \& Matteucci, F. 2018, Nature, 558, 260 